\title{
Composition of Languages Embedded in Scala
}

\author{
Seyed H. HAERI (Hossein) \\ Université catholique de Louvain, Belgium \\ hossein.haeriducl.ac.be
}

\author{
Paul Keir \\ University of the West of Scotland, UK \\ paul.keireuws.ac.uk
}

\begin{abstract}
Composition is amongst the major challenges faced in language engineering. Erdweg et al. offered a taxonomy for language composition. Mernik catalogued the use of the Language Definitional Framework LISA for composition sorts in that taxonomy. We produce a similar catalogue for embedded language engineering in Scala.

We begin with techniques that are not specific to Scala. They are applicable in any host language with a module system and support for higher order functions. We, then, present two more techniques to examine Scala-specific language engineering. Interestingly enough, even though dealing with embedded languages, in terms of lines of code, our material is of comparable length to its LISA counterpart. Our work lends insight into Scala's serviceability for composition, as a host for embedded language engineering.
\end{abstract}

\section{INTRODUCTION}

a) Language composition is a piece of reality!: Everyday, there are new programming languages that are born by combining ideas from older languages. Inspiration aside, that is an act of composition in many cases. For example, roughly put, Scala adds functional programming and ML modules with mixin composition to Java; which, in return, is $\mathrm{C}++$ without pointers; which, in return, is $\mathrm{C}$ with OOP.

The taxonomy of Erdweg et al. [1] suggests a terminology and notations for describing such compositions. According to them, one can formalise our Scala description as:

$$
\text { Scala } \approx \mathrm{C} \triangleleft \mathrm{C}++\triangleright \text { Java } \triangleleft(\text { MLModule } \uplus \text { Mixin })
$$

b) Observations from Chemistry: Consider the reaction:

$$
\mathrm{H}_{2} \mathrm{SO}_{3}+2 \times \mathrm{NaOH} \longrightarrow 2 \times \mathrm{H}_{2} \mathrm{O}+\mathrm{Na}_{2} \mathrm{SO}_{3}
$$

In Chemistry, two key ingredients for success in the study of such equations are: $\left(\mathbf{C I}_{1}\right)$ the availability of substances as the subjects of study, and, $\left(\mathbf{C I}_{2}\right)$ knowledge about how to perform a desirable composition. In reaction (2), for instance, both substances $\mathrm{H}_{2} \mathrm{SO}_{3}$ and $\mathrm{NaOH}$ need to be available. One also needs to know how to double $\mathrm{NaOH}$ for the equation balance to be right. Also, how to add $\mathrm{NaOH}$ to $\mathrm{H}_{2} \mathrm{SO}_{3}$ (like the rate of addition, proper temperature, etc.) needs to be known.

c) Programmatic Availability \& Composition: The study of formulae like equation (1) determines the precise relative position of languages. Using the outcome, one would be able to add, for example, what is missing in equation (1) so that the " $\cong$ " can be replaced by an "=". One would also gather that the left-out "FP $\uplus$ " is necessary right before MLModule for the balance to be right. Such manipulations are similar to adjusting coefficients in reaction (2) to obtain a balance. Similar to Chemistry, two key ingredients become noticeable here: (PLI $\mathbf{I}_{1}$ ) programmatic availability of programming languages themselves and their belongings as the subjects of study, and, $\left(\mathbf{P L I}_{2}\right)$ knowledge about how to programmatically obtain desirable language compositions.

By the time of this writing, (mainstream) languages are next to inaccessible as programmatic entities. The study of programmatic language composition, nonetheless, can be conducted independently using, say, contrived languages. That is how this paper tries to gain $\left(\mathbf{P L I} \mathbf{I}_{2}\right)$

d) Contributions: We demonstrate three techniques for composing languages embedded in Scala. The first (Section II) is applicable in any host language with a module system and support for higher order functions. The second (Section III) is based on Lightweight Modular Staging (LMS) [2]. And, the third - which is also a new solution to the Expression Problem (EP) [3], [4], [5] - employs (possibly restricted) abstract types. The trick in our third technique is promoting the cases of Algebraic Data Types (ADTs) into their own ADT-parameterised standalone components. We showcase each technique using the example compositions of Mernik [6]. We, then, compare the three techniques for their success in addressing the EP concerns (Section V). A discussion about the related work also comes at Section VI.

e) Coding Conventions: This paper assumes familiarity with Scala. For each showcase, the syntax and semantics come in separate packings called syntax and semantics, respectively. Due to space restrictions, in our code, the name of the showcase is only appended as a comment to the end of the first line of the respective syntax or semantics. For the same reason, our code is also otherwise unusually compressed. Whilst the showcases are referred to in the prose in CamlCase, their respective Scala package (containing the showcase's syntax and semantics) is named like_this or abbreviated as $1 t$.

\section{SCALA-UNSPECIFIC}

Erdweg et al. catalogue five different ways languages can be composed: language extension, language restriction, language unification, self-extension, and extension composition. Mernik offers simple DSLs to showcase those ways in LISA [7]. In this section, we employ Mernik's simple DSLs for the same purpose, albeit in Scala. 


\section{A. Language Extension}

A base language $B$ is said to be extended to a language $E$ when the description of $B$ is amended with a description fragment to get $E$. Erdweg et al. denote that by $B \triangleleft E$. Consider the language Robot below (packaged under the name robot in Scala) for a robot arm that takes commands for moving one unit to either of the four $2 \mathrm{D}$ directions. The semantics of Robot involves updating the arm's position (recorded in terms of the $x$ and $y$ coordinates) based on the commands (lines 11 to 16 ).

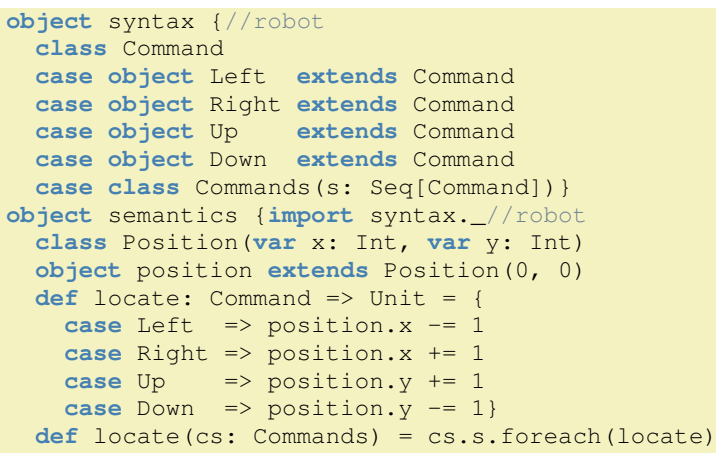

Robot is extended to RobotTime (the robot_time package) by adding to the semantics, i.e., Robot $\triangleleft$ RobotTime:

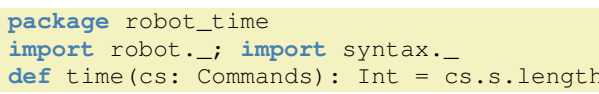

Assuming that executing each command takes one time unit, the total time required for a set of commands is the size of the set. The method time in line 3 above adds that piece of semantics to Robot to get RobotTime. whereas Commands ( Right, Down, Down) in Robot has only got the semantics $x=1, y=-2$, it also has the semantics $t=3$ in RobotTime. (The coordinates are obtained by locate in line 16 of robot and the timing by line 3 of robot_time.)

Here is a difference between our implementation of Robot- ${ }^{10}$ Time and that of Mernik: The latter is done in LISA: a Language Definitional Framework (LDF) that combines OOP 13 with Attribute Grammars (AGs) [8], [9]. As such, LISA's ${ }_{15}^{14}$ counterpart for time has to visit all the grammatical rules ${ }^{16}$ in Robot to attribute the new piece of semantics to them. On ${ }_{18}^{17}$ the contrary, Scala gave us the joy of simply equating time by ${ }^{19}$ the number of the commands, regardless of the grammatical rules involved.

\section{B. Language Restriction}

A base language $B$ is said to be restricted to a language $R$ when certain parts of the $B$ 's features are removed upon transition to $R$. This is denoted by $B \triangleright R$. A typical usage of that is when a language is narrowed to a core of it. That is, certain parts of the base syntax are cancelled into combinations of other base syntactic parts that are deemed to be equivalent. For example, both GPH [10] and Utrecht HASKELL [11] are developed like that.
The language RobotPositive below (packaged under robot_positive) restricts Robot to only Up and Right commands. (Technically, the object syntax below is not required. Yet, we retain it for completeness.)

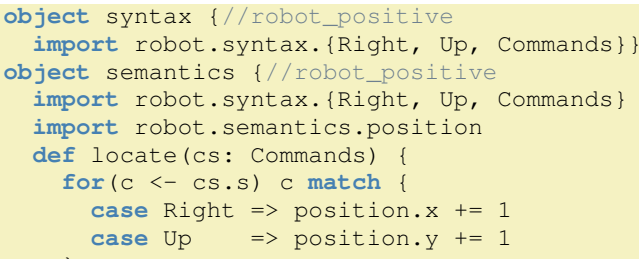

Any attempt to use the expression in the previous section under RobotPositive will fail to compile for the availability of Down in it, which is absent in RobotPositive. On the other hand, Commands (Right, Up, Up) has the semantics $x=$ $1, y=2$ under RobotPositive.

\section{Language Unification}

Erdweg et al. say two languages $L_{1}$ and $L_{2}$ are unified to $L$ when both $L_{1}$ and $L_{2}$ make sense independently from one another and from $L$ (as the composition's outcome). Furthermore, in $L$, neither $L_{1}$ nor $L_{2}$ should be dominated by the other so that a concept of equity prevails in the composition. The notation is $L=L_{1} \uplus_{g} L_{2}$, where $g$ is the so-called glue code required for the composition.

Having seen the language Robot, we now consider the language ExprAdd (packaged under expr_add): a simple ADT with two cases for natural numbers and addition.

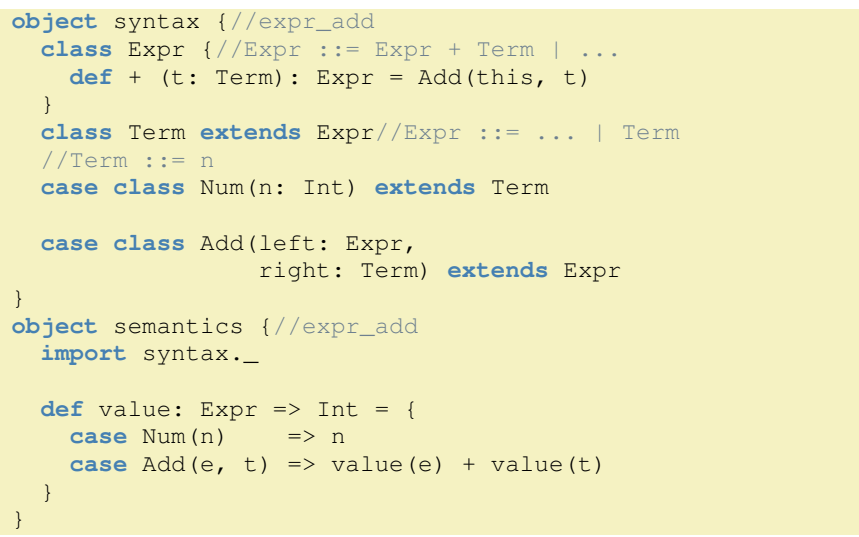

Using value in line 15 above, one obtains the semantics 5,12 , and 6 for the expressions Num (5), Num (10)+ Num(2), and Num(1) + Num(2) + Num(3), respectively.

The language RobotUniExprAdd below (packaged under robot_uni_expr_add) unifies Robot and ExprAdd by allowing the robot arm to take commands for moving as many units to either of the four directions as the corresponding ExprAdd argument evaluates to. As such, Commands (Right (Num(5)), Up (Num(2) + Num(10)), Up (Num(2) $+\operatorname{Num}(2)+\operatorname{Num}(2))$, Down (Num(4))) has the semantics $x=5, y=14$. Check locate in line 17 below. 


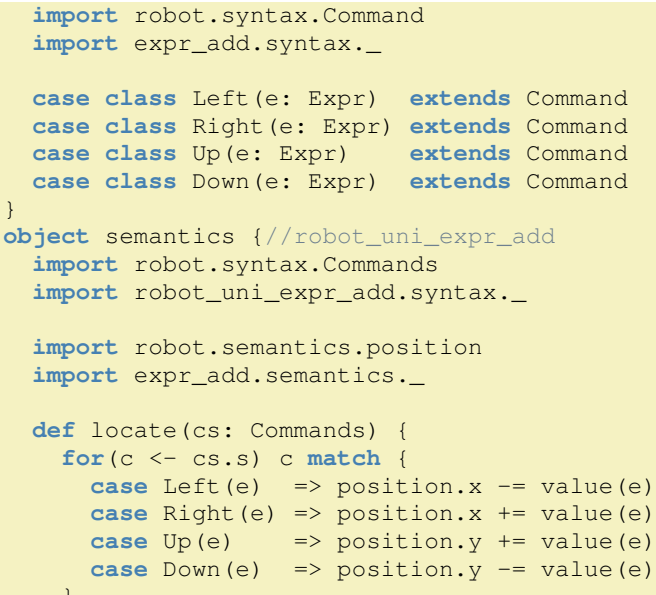

\section{Self Extension}

This is the situation when the description of a language $L$ itself is used for extending it. Typically, embedded DSLs selfextend their host language. For example, all the languages we present in this paper self-extend Scala.

Like Mernik, we believe that demonstrating self extension takes much more than the volume of a single research paper. This is because bootstrapping a language $L$ to the level where it can handle self extension is already more involved than that volume. Hence, we too drop demonstration of self extension.

\section{E. Extension Composition}

Extension composition is when (both or at least one of) the language descriptions that are to be composed are themselves compositions of other language descriptions. As such, extension composition can be regarded as higher order composition. Six combinations of extension and unification are possible (three distinguished by Mernik):

1) Double-Unification $(\uplus \uplus): L_{1} \uplus_{g}\left(L_{2} \uplus_{h} L_{3}\right)$.

2) Double-Extension $(\triangleleft \triangleleft)$ : $B \triangleleft E_{1} \triangleleft E_{2}$.

3) Extension by a Unification $(\triangleleft(\uplus))$ : $B \triangleleft\left(L_{1} \uplus L_{2}\right)$.

4) Extension of a Unification $((\uplus) \triangleleft)$ : $\left(L_{1} \uplus L_{2}\right) \triangleleft E$.

5) Unification with an Extension $(\{\uplus,(\triangleleft)\}): L \uplus(B \triangleleft E)$ or $(B \triangleleft E) \uplus L$. Note the symmetry.

We now consider each combination.

1) Double-Unification $(\uplus \uplus)$ : To that end, we begin by presenting Mernik's language Dec (packaged under dec) in Scala. Dec enables the programmer to bind a set of variables to integer constants.

\section{object syntax \{//dec
case class ConstDefList(ds: Map[String, Int]) \}}

Unsurprisingly, the (Scala-automatic) semantics of ConstDefList ("a" -> 5, "b" $->10$ ) is then $\{a \mapsto 5, b \mapsto 10\}$.

With that, we illustrate the first class of Mernik's extension compositions using RobotUniExprAddUniDec (packaged under rueaud). As suggested by its name, this language is (Robot $\uplus$ ExprAdd $) \uplus$ Dec. The Robot $\uplus$ ExprAdd portion is already presented. See robot_uni_expr_add in Section II-C. We now show how to obtain the remaining unification.

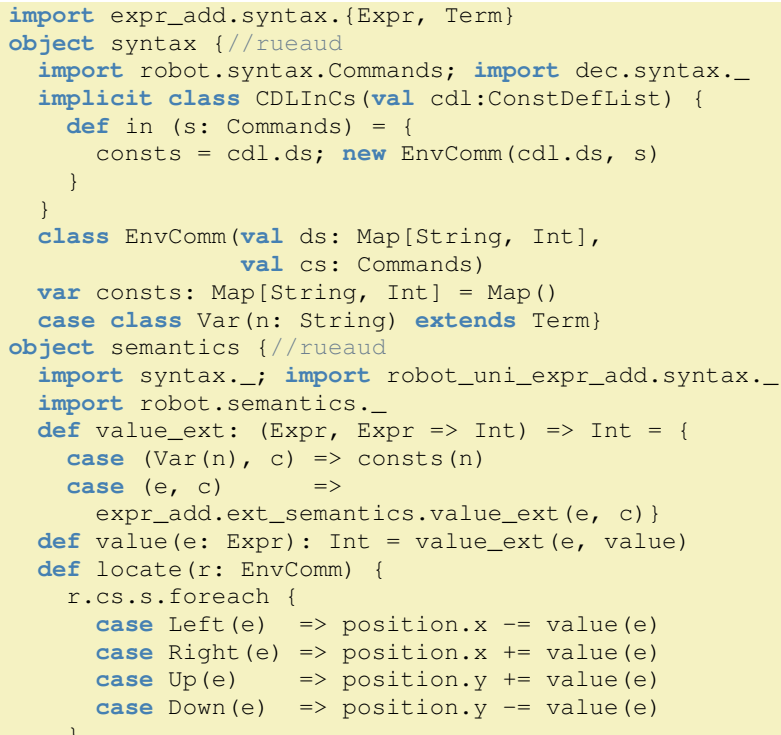

rueaud.syntax aims at reusing the former language descriptions as they are. To that end, it takes a pimp my library approach [12] on trying to implicitly (lines 4 to 10 above) give instances of dec.ConstDefList the extra feature of being followed by commands possibly referring to the declarations. Such declarations followed by expressions are then instances of EnvComm. The variable consts (line 11) is where the processed declarations are stored. The new ADT case Var (line 12) is for looking up the value a name is bound to. rueaud legitimises commands for moving the robot arm as many units as a pertaining expression evaluates to (lines 23 to 26). Note that, because of Var, those expressions can refer to declarations as well. All that together gives ConstDefList ("a" -> 5, "b" -> 10) in Commands (Right(Var(" a")), Up (Num(2) $\operatorname{Var}(" \mathrm{~b} ")), \operatorname{Down}(\operatorname{Num}(4)))$ the semantics $x=5, y=8$ in RobotUniExprAddUniDec.

Instead of reusing expr_add.semantics.value, the rueaud.semantics.value method uses the method expr_add.ext_semantics.value_ext, which will be explained shortly. This is because the former is closed on the set of ADT cases it can handle. Hence, we resort to the following extensible semantics of ExprAdd:

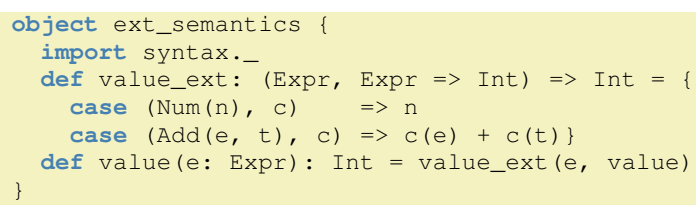

In the fashion of $\gamma \Phi C_{0}$ [13], value_ext above takes a continuation argument $\mathrm{c}$ (line 3 ), which caters postponing the closing time until the appropriately complete shape [14] of the ADT is known (line 6 above for expr_add and line 20 for rueaud). As such, extending RobotUniExprAdd to RobotUniExprAddUniDec here involves manipulating the former. See Section $\mathrm{V}$ for more.

2) Double-Extension $(\triangleleft \triangleleft)$ : The idea in RobotTimeSpeed below (packaged under robot_time_speed) is to enable 
the user to instruct the robot arm with the speed for its 10 subsequent moves, until further notice. It adds a pertaining command to RobotTime to obtain Robot $\triangleleft$ RobotTime $\triangleleft 1$ RobotTimeSpeed.

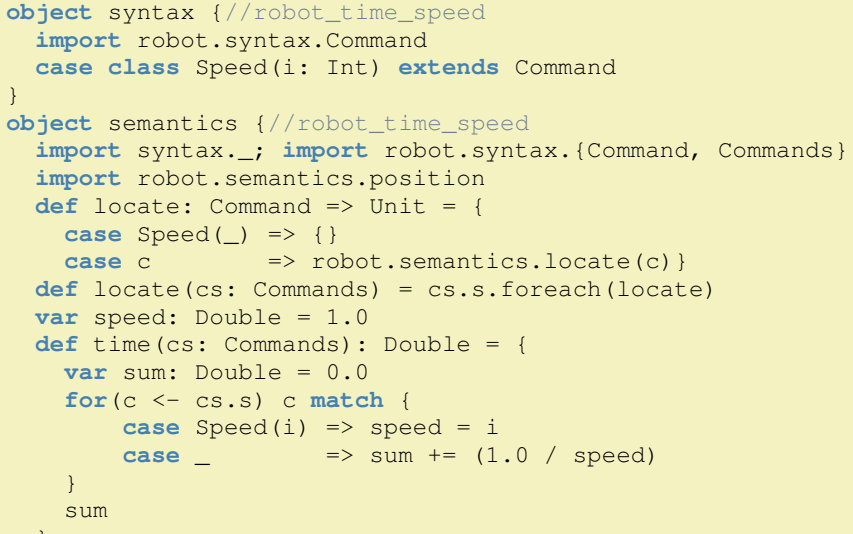

The new command for altering speed is speed in line 3 above. This new command has no impact on the arm's position, as manifested in line 9 . It is in the time calculation where, once used, the related variable (i.e., speed in line 12) is updated accordingly (line 16) and taken into consideration for subsequent commands (line 17). Commands (Up, Speed (2), Right, Left) has the semantics $x=1, y=0, t=2$ in RobotTimeSpeed.

3) Extension by a Unification $(\triangleleft(\uplus))$ : We now demonstrate RobotExtExprAddUniDec $=$ Robot $\triangleleft($ ExprAdd $\uplus$ Dec $)$. We begin by ExprAddUniDec (packaged under eaud):

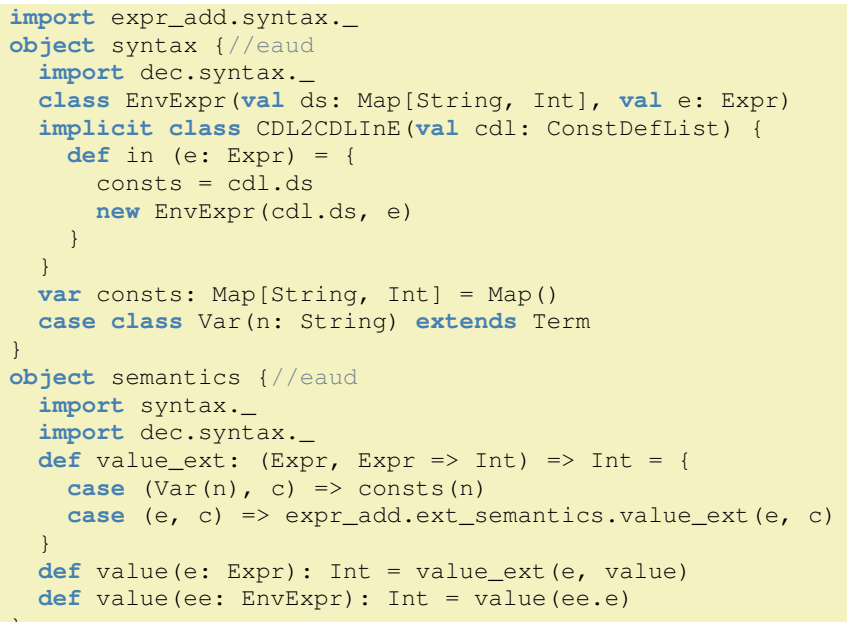

eaud is similar to rueaud in Section II-E1 and we drop further explanation. RobotExtExprAddUniDec below (packaged under reeaud) tries to make use of eaud.

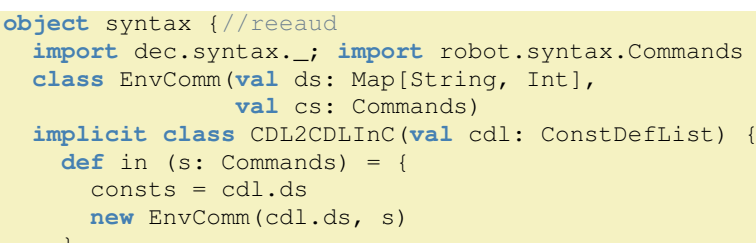

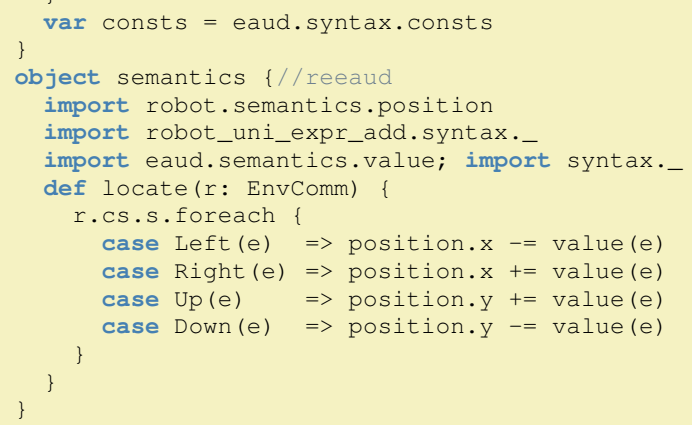

Here are the few idiosyncrasies of reeaud: Firstly, reeaud fails to reuse most of the syntactic facilities of eaud. This is because the former employs declarations followed by commands, whereas the latter employs declarations followed by expressions. In line 11, nevertheless, consts is reused. Secondly, even though RobotExtExprAddUniDec = Robot $\triangleleft \ldots$, in reeaud.semantics, we do not reuse robot.syntax. On the contrary, in line 15 , it reuses the syntax of robot_uni_expr_add (for RobotUniExprAdd). This is because, in Robot, it is only possible to move the arm one unit to either direction. The Scala syntax for those two pieces of (embedded) syntax cannot coexist side by side. See Section III-A2 for more.

reeaud.semantics.locate is similar to rueaud.semantics.locate. In RobotExtExprAddUniDec, ConstDefList ("a" -> 5, "b" -> 10) in Commands ( $\operatorname{Right}(\operatorname{Var}(" \mathrm{a} ")), \operatorname{Up}(\operatorname{Num}(2)+\operatorname{Var}(" \mathrm{~b} "))$, Down ( Num (4))) has semantics $x=5, y=8$.

As pointed out by Mernik, so long as functionality is the only concern, RobotUniExprAddUniDec $\equiv$ RobotExtExprAddUniDec. The difference, both in LISA and Scala, is in the language descriptions, and the combinations by which they are obtained. Unlike its LISA counterpart, nonetheless, obtaining RobotExtExprAddUniDec in Scala involves intermediate material that is not reused in the final product.

4) Extension of a Unification $((\uplus) \triangleleft)$ : RobotUniExprAddExtRobotTime below (packaged under rueaert) extends RobotUniExprAdd (Section II) by a timing facility. The time required for carrying out a command of moving in one direction equals what the pertaining expression evaluates to (lines 9 to 12). The method time below is a simple fold operation on the given sequence of commands, based on that explanation. RobotUniExprAddExtRobotTime $=($ Robot $\uplus$ ExprAdd $) \triangleleft$ RobotTime.

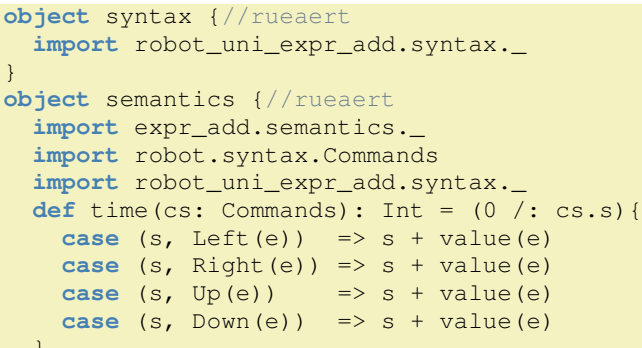


Commands (Right (Num(5)), Up (Num(2) + Num(10)), $\mathrm{Up}(\operatorname{Num}(2)+\operatorname{Num}(2)+\operatorname{Num}(2)), \operatorname{Down}(\operatorname{Num}(4)))$ has the semantics $x=5, y=14, t=27$ in rueaert.

5) Unification with an Extension $(\{\uplus,(\triangleleft)\})$ : Take RobotUniExprMul = Robot $\uplus$ ExprMul, where ${ }^{22}$ ExprAdd $\triangleleft$ ExprMul. The language ExprMul extends ${ }^{24}$ ExprAdd by a new ADT case for multiplication (Mul). What is unique about ExprMul amongst the visited extension combinations is that, upon extension, it changes the syntactic categories of the ADT cases it borrows from ExprAdd. (And, in fact, it also provides a new syntactic category, i.e., Factor.) As presented in Section III-B, this can impose a great deal of complexity when language extension is implemented using inheritance. Here is ExprMul (packaged under expr_mul).

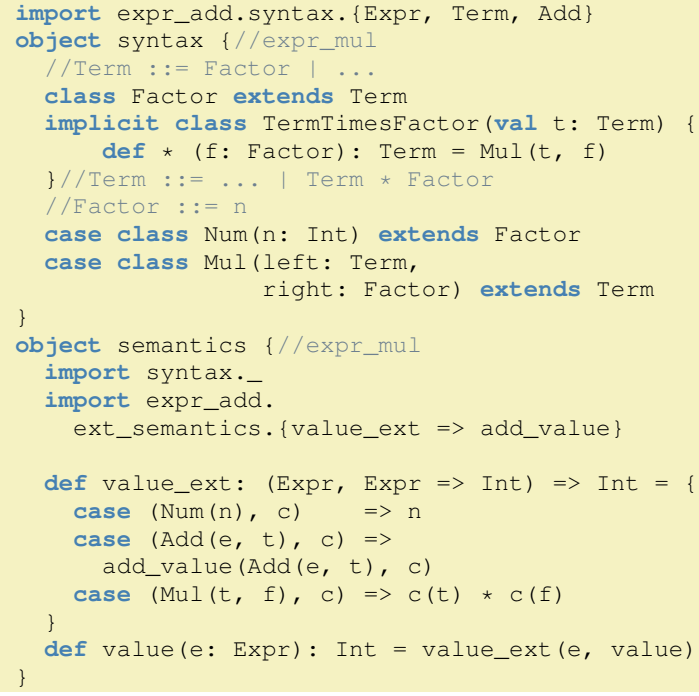

In line 1, ExprMul imports the syntactic entities it borrows from ExprAdd: the ADT case Add and the syntactic categories Expr and Term. It then introduces its new syntactic category Factor in line 4. Next, in lines 5 to 7, it provides the syntactic sugar for multiplication. Note how it, afterwards, declares numbers to now be of the category Factor - as opposed to Term in expr_add.syntax. The rest of expr_mul should be straightforward except for the Scala syntax of lines 15 to 16. Those lines abbreviate expr_add.ext_semantics .value_ext to add_value in expr_mul.semantics. In line 21, expr_mul reuses add_value for the solo ADT case that it borrows from expr_add, i.e., Add.

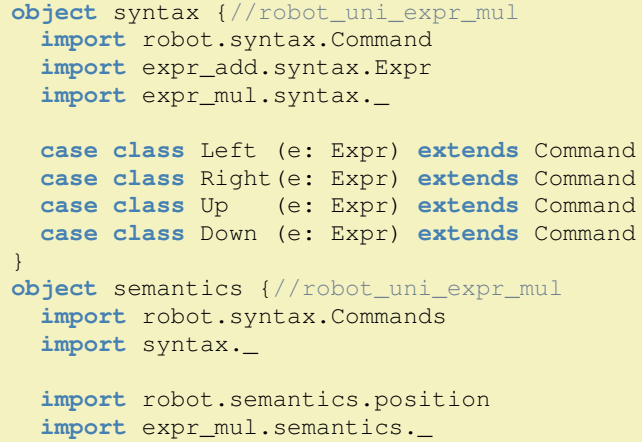

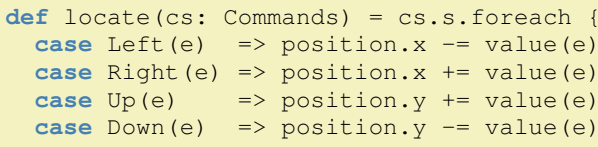

The above implementation of RobotUniExprMul (packaged under robot_uni_expr_mul) takes tightly after RobotUniExprAdd (in Section II-C). We, therefore, do not provide a dedicated walk-through. Commands (Right (Num (5)* Num (2)), Down (Num(4)+Num(2)* Num(3))) has the semantics $x=10, y=-10$ in robot_uni_expr_mul.

\section{F. Language Specific?}

To investigate the extent to which Scala-specific language features impact upon our design, we intend also to compare against realisations in other languages. To this end, we have prepared a $\mathrm{C}++$ implementation which adopts the Scala approach outlined so far. Respecting the dynamic polymorphism of the Scala original, the $\mathrm{C}++$ implementation utilises shared_ptr smart pointer to manage the memory allocation and runtime typing of expressions; allowing the vector container member object of the Commands class to store different expression types. User-defined integral and string literals also allow a notably concise syntax for the Num and Var instantiations; e.g., Commands $\left\{\right.$ Right $\{$ "a"_s $\}, \operatorname{Up}\left\{2 \_n+\right.$ "b"_s $\}$, Down $\left.\left\{4 \_n\right\}\right\}$. Future work will explore this further.

Note that we are keen in the solution of this section not to employ Scala's built-in open recursion. Due to unrelated reasons, however, Scala compilers might still employ open recursion internally to compile our code. Nonetheless, our code does not require that Scala idiosyncrasy. Testimony to that lack of requirement is our $\mathrm{C}++$ code. Note that whilst open recursion is automatic in Scala, in $\mathrm{C}++$, one needs to explicitly use "this->" for the late-binding of open recursion.

\section{LMS-BASED}

Rompf and Odersky [2] coin Lightweight Modular Staging (LMS) for Polymorphic Embedding [15] of DSLs in Scala. They employ a fruitful combination of the Scala features detailed in [16] that, as a side-product, offers a very simple yet effective solution to EP. In this paper, we use LMS for that EP solution. The essence of LMS is the use of Scala traits for extensibility and super calls for reuse. With their mixin nature, Scala traits can extend one another, enjoying the benefits of inheritance. In particular, an ADT can be inherited upon trait extension. But, the heir trait can also add its own new ADT cases. On top of that, super calls enable reusing methods on the cases of the original ADT. Whereas the new cases can be handled by the same method, albeit overridden by the heir trait.

In the package eaud below (for ExprAddUniDec), for implementing both the syntax and semantics, traits are used - as opposed to objects in Section II. Instead of importing members from other languages, it now extends those other languages to acquire the same members via inheritance. In 
Scala terms, eaud.syntax is, for instance, said to be mixing in expr_add.syntax and dec.syntax, in line 1 below.

In line 4, then, eaud. semantics overrides value. In line 5, it handles the new ADT case eaud. synt ax introduces. All those other ADT cases that eaud inherits are, in line 6, relayed to the upper levels of inheritance.

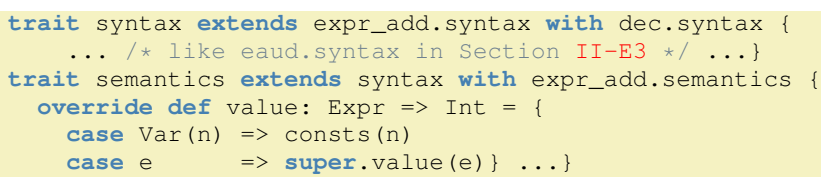

This is how LMS facilitates both simplicity and extensibility. (Note that we needed not to resort to value_ext.)

LMS has been successfully employed for languages in a multitude of applications. For the benefits of LMS, the reader is invited to consult those works. Given that we did not come to observe new benefits, we will not get into that here. We rather dedicate this section to the difficulties we faced over employing LMS for embedded language composition.

\section{A. Minor Difficulties}

The two categories of minor difficulties we faced relate to language restriction (Section III-A1) and clashes occurred between names upon composition (Section III-A2).

1) Language Restriction: Upon extension, the programmer is usually provided with no means for acting selectively on the members to be inherited. When mixing traits too, all the (public or protected) members get inherited automatically. Hence, with inheritance being the means for language composition, language restriction is not possible. That enforces import as the fallback. With the use of traits, the mechanics is, however, more involved than Section II. Because traits are abstract, one needs to materialise them first (line 2 below), and only then, they can be imported from (line 3 ).

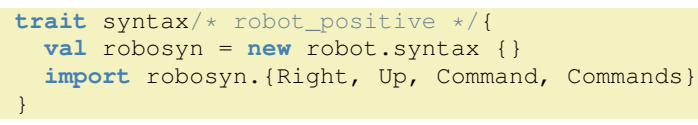

Even though LISA also employs inheritance for language composition, this difficulty does not arise there. The reason is as follows: Being also an AG system, (subject) language semantics is specified in LISA by traversing the concrete syntax. On the other hand, leveraging its OOP, LISA allows the heir language to override the parent language's concrete syntax. As a result, language restriction is also possible in LISA via inheritance.

One final related comment: In our experience, enforced imports like those required for language restriction were not exclusive to that way of language composition. In fact, in a good number of other occasions, the languages do make selective use of one another. That, on its own, was not a knotty problem. It, however, requires increasingly more care when it comes to interplay with hierarchies of languages and the relevant Scala mixins.

Note that imported names (like those in line 3 above) do not get inherited but the respective materialised traits (like robosyn in line 2 above) do. Such imports can be required on several occasions down the hierarchy. In the case of unification, however, where the multiple inheritance nature of mixins is employed, an extra override might also be enforced to disambiguate duplicated names across the meeting two hierarchies. See Section III-B for more.

2) Name Clash: Recall from Section II-E3 that RobotExtExprAddUniDec $=$ Robot $\triangleleft$ (ExprAdd $\uplus$ Dec $)$. In an LMS-based implementation of RobotExtExprAddUniDec, therefore, one would naturally want to implement rueaud. semantics as follows:

trait semantics extends rueaud.syntax with

robot.semantics with eaud.semantics $1 / /$ ruea

. * locate like Section II-E1 */ ...

That is, however, not possible. The error message is: "object Left is not a case class, nor does it have an unapply /unapplyseq member." The problem is that, even though Left is inherited from robot, in locate, Scala would not be able to match it using the syntax Left $(e)$. The available constructor and extractor of Left take no arguments. Moreover, overloading that syntax is not possible. This is because Scala desugars both case classes and case objects to objects with unapply (or unapplyseq) methods. Objects, on the other hand, are final, banning any later manipulation. To proceed, one needs to use robot_uni_expr_add.semantics in return of robot. semantics.

The problem is harder to diagnose for RobotUniExprAddExtRobotTime. Recall from Section II-E4 that RobotUniExprAddExtRobotTime $=($ Robot $\uplus$ ExprAdd $) \triangleleft$ RobotTime. For the attempt

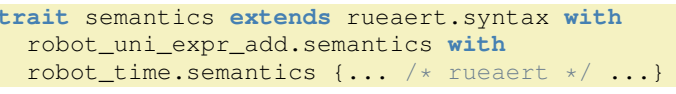

even when one employs robot_uni_expr_add.semantics instead of robot.semantics, one gets an error - this time, regarding the composition itself: "overriding object Left in trait syntax; object Left in trait syntax cannot override final member." The problem here is with robot_time being an extension to robot, bringing the case object Left into the mix with that of robot_uni_expr_add that takes an argument.

\section{B. Major Difficulties}

The difficulties we spoke about in the previous subsection were not particularly acute in that not many circumvention attempts would fail for them. In this section, we will report a multi-staged combat with an acute difficulty we faced. In short, the combat was against the combination of Scala's pathdependant typing and intervention of concrete syntax.

The contents of this section might look too specific to Scala. They are not. Scala's path-dependant typing is just one way to foster family polymorphism [17] (as opposed to lightweight family polymorphism [18]). The familiar reader will figure out that the same problem is likely to emerge in every host language that embraces family polymorphism.

Given that ExprMul is a direct extension to ExprAdd, one's first guess would be: 
trait expr_mul.syntax extends expr_add.syntax $\{\ldots\}$

That is, however, not possible because, then, Num cannot be overridden. Recall from Section II-E5 that ExprMul changes the syntactic category of Num. But, even an attempt like those in Section III-A1 for the syntax

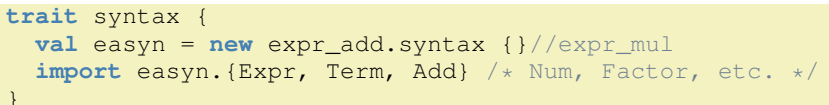

would still cause failure for the semantics.

trait expr_mul.semantics extends syntax with expr_add.semantics $\{\ldots\}$

Here is the error message: "overriding object Num in trait syntax; object Num in trait syntax cannot override final member." This is because of the clash between the Num of such a expr_mul.syntax and expr_add.semantics. See Section III-A2 for an explanation on similar error messages.

Now, let us suppose for the sake of argument that the semantics too selectively imports the ADT cases:

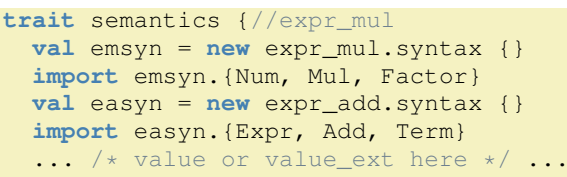

Recall that ExprMul adds the ADT case Mul to ExprAdd. To reuse - à la LMS - the ExprAdd semantics whilst also handling the new ADT case, one may (mistakenly) try:

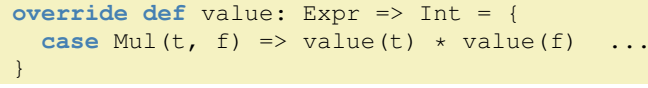

But, that will not type-check because of path-dependant typing interference: Expr in value's signature is different from Expr that Mul inherits from. Here is the error message for line 2 above: "constructor cannot be instantiated to expected type; found: semantics.this.emsyn.Mul required: semantics.this.Expr." Even worse: An attempt for reusing the semantics of the only ADT case that remains intact over the move from ExprAdd to ExprMul using value_ext

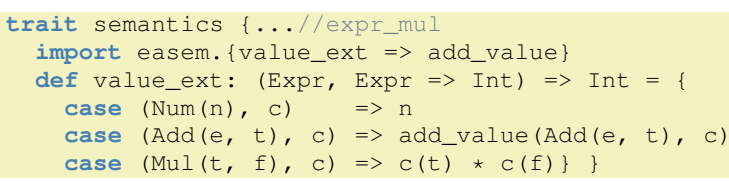

will again fail due to path-dependant typing. The error message for line 5 above is: "type mismatch; found: semantics.this .easyn. Add required: semantics.this.easem.Expr."

Given that expr_mul.semantics is to reuse pattern matching of expr_add.semantics, the former is also bound to the types - here , ADT cases - of the latter. In order to prevent the path-dependant clashes, thus, the only way forward seems to be for both expr_mul.syntax and expr_mul. semantics to import types of expr_add.semantics. This is, of course, very unnatural for the former.

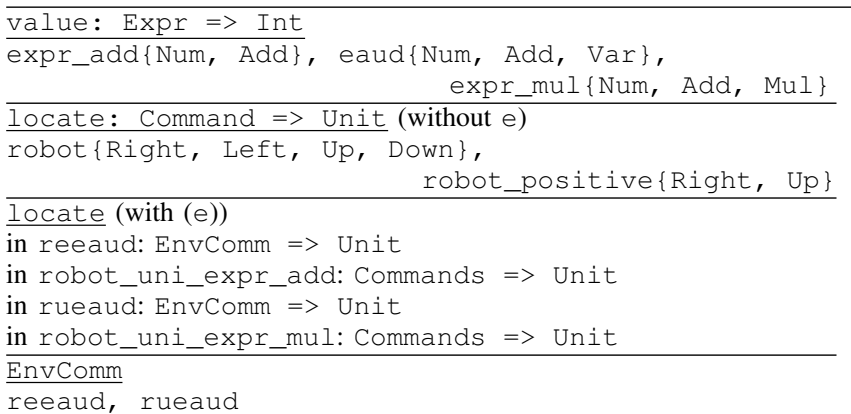

Fig. 1: Duplicate Entities in Sections II and III

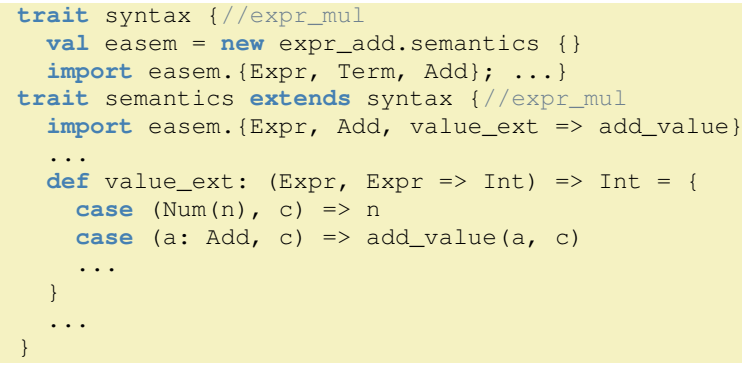

Still, if not done craftily enough, path-dependant typing can be an impediment. Replacing the line 9 above with

case (a a Add (_, _ ), c) $=>$ add_value $(\mathrm{a}, \mathrm{c}$ ) will fail to type-check because $a$ is considered to be of type this.Add; whereas, add_value accepts an easem.Expr. The unsightly circumvention would be:

case (a e Add (_, _ ), c) => add_value (a.

asInstanceof [easem.Expr], c.asInstanceof [easem . Expr $=>$ Int]).

We would like to remind that all the difficulties illustrated in this section were only experienced in the presence of manipulation in the syntactic categories upon extension. Syntactic categories are often used for dealing with concrete syntax. Semantics, on the other hand, inputs abstract syntax. The following section presents a solution that disassociates concrete syntax from abstract syntax. It applies the LMS at the abstract syntax level, and, hence, independently of the concrete syntax that varies across languages. That design sets the different languages free on engineering their syntactic categorisation whilst enjoying the benefits of LMS.

\section{REFACTORING}

The previous two sections were developed as if the guest language implementer was not aware in advance of the next guest languages and the upcoming combinations. We also maintained a backward compatibility policy in that we did not touch the older languages as we proceeded. Refactoring, however, is common in everyday software development.

Refactoring can have a variety of meanings, depending on the target and the methods used [19]. Here, we do not plan extensive refactoring. We only focus on duplicate elimination in the fashion of the extract superclass method [19, §12.6]. Fig. 1 lists a number of duplicates in Sections II-III. 
We notice that the method value is duplicate in expr_add, eaud, and expr_mul. More precisely, the ADT cases Num and Add - which are, basically, inherited from expr_add are handled thrice in the codebase. As will be shown in this section, we gave value its own abstraction.

We also notice that the method locate is present in two sets of language descriptions: in (i) robot and robot_posit -ive (when the four direction commands do not take arguments); and, in (ii) reeaud, robot_uni_expr_add, rueaud, and robot_uni_expr_mul (when the four direction commands do take arguments). Each of those sets constitutes a candidate for refactoring. Finally, EnvComm is common between reeaud and rueaert - constituting yet another refactoring candidate. Although we have indeed refactored the candidates of this paragraph as well, we will not include their demonstration in this paper. The interested reader can look them up in our online codebase.

Let us now focus on refactoring the first row of Fig. 1. (Refactoring the other rows of Fig. 1 is done similarly.) Here is a succinct summary of actions to be taken: The idea is a combination of LMS and Component-Based Mechanisation [20], [21], [13]. We parameterise the ADT cases Num, Add, Var, and Mul by the language description and perform their semantics evaluation independently of the language description. We pack the two former cases - namely, Num and Add that are common between all the items in the first row of Fig. 1 - together in a trait. Then, we extend that trait for Var and later for Mul, both à la LMS. Finally, the concrete language descriptions only get to mix the respective abstract descriptions. The elaboration follows.

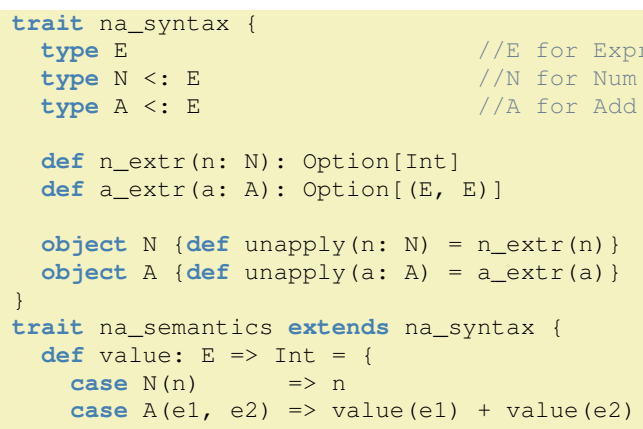

In the trait na_syntax above, the abstract type $\mathrm{E}$ (in line 2) is a language-independent representation for the expression type of a guest language. Such a guest language can be an item in row 1 of Fig. 1 or any similar language with integer arithmetics that at least contains integral literals and addition. Given that ADTs are implemented in Scala using plain inheritance, two more language-independent abstract types have been employed that are announced to be extending $\mathrm{E}$. Those are $\mathrm{N}$ for Num and $\mathrm{A}$ for $\mathrm{Add}$, in lines 3 and 4.

Because $\mathrm{N}$ and $\mathrm{A}$ are supposed to later be instantiated to the respective cases of an ADT, they are expected to come with the Scala matching syntax, like those in lines 14 and 15. The Scala machinery for enforcing availability of the desirable matching syntax requires a discipline in coding that is slightly tricky. The discipline involves, for each ADT case abstract type, inclusion of a same-named (singleton) object called companion object - that ships, then, with an extractor, i.e., an unapply method of the right type signature. The actual duty of the extractor is relayed to an abstract method, to be enforced to every guest language that implements na_syntax. For $\mathrm{N}$, for instance, that duty is on n_extr in line 6. The Scala signature of n_extr means that, if matching N succeeds, it would be initialising an argument of type Int. All that wiring enables the method na_semantics . value to handle the semantics of Num and Add.

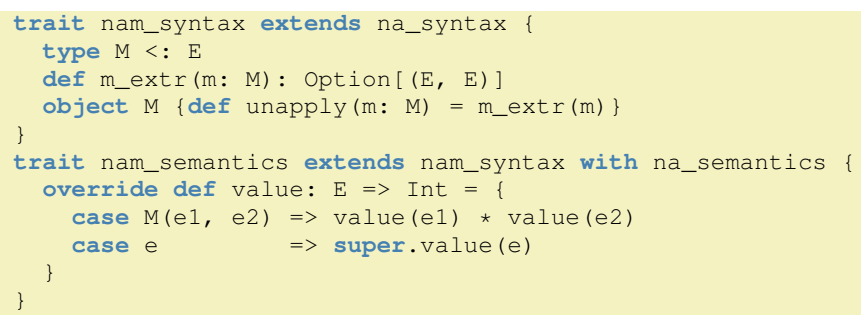

The trait nam_syntax adds the abstract type $M$ (in line 2 above), which corresponds to Mul. It also provides the Scala matching syntax in lines 3 and 4. The trait nam_semantics reuses (à la LMS) what is already implemented by na_semantics by performing a super call on the relevant ADT cases (line 9)

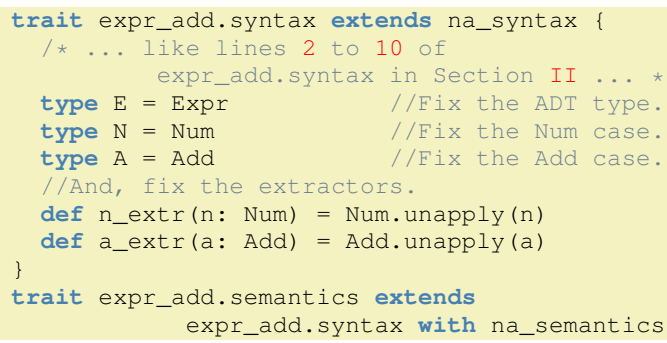

In addition to working out the Section II concrete syntax, the trait expr_add.syntax above, now is required to provide evidence on it indeed having ADT cases for integral literals and addition. That, again involves some slightly tricky discipline consisting of two steps. First, in lines 4 to 6 , the concrete counterparts for the abstract (ADT case) types in na_syntax are fixed. Second, in lines 8 and 9 the extractors promised to na_syntax are fixed.

Recall from expr_add.syntax of Section II that Num and Add are both case classes. Scala actually desugars case classes to normal classes in addition to companion objects with the right-typed unapply methods. That is why we can use Num . unapply and Add. unapply off-the-shelf.

Nothing more remains for expr_add.semantics to do except inheriting its (abstract and concrete) syntax from expr_add.syntax and its semantics from na_semantics.

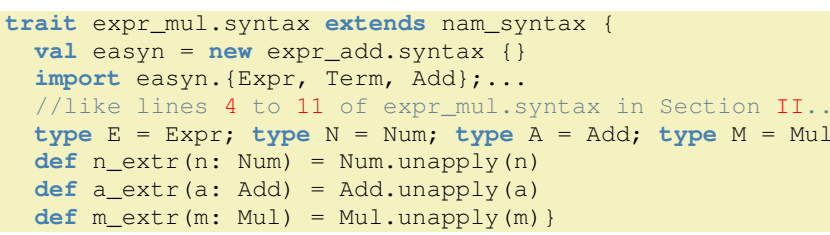


trait expr_mul.semantics extends

expr_mul.syntax with nam_semantics

Implementing ExprMul, in this fashion, is similar, as demonstrated above. It only is that, like in Section III, our use of traits instead of objects in favour of LMS imposes instantiation of the trait expr_add.syntax (line 2) before importing the desirable concrete syntax items (line 3).

\section{Remarks}

na_semantics is similar to how one defines the semantics of Num and Int using Modular Structural Operational Semantics (MSOS) [22]. In MSOS, the semantics of a component is defined exclusively in terms of the relevant language elements - making it ignorant about all other language elements. na_semantics only concerns Num and Int, and, is ignorant about other language elements. $\gamma \Phi C_{0}$ [13] describes that as: "client na_semantics $<F \triangleleft$ Int $\oplus$ Num $>\{\ldots\}$," where $F$ is the family parameter of na_semantics. In words, that reads: A family $\Phi$ to be substituted for $F$ needs at least to have components Int and Num (or their equivalents) in its mix.

From another language theoretical viewpoint, na_syntax and na_semantics are both type classes [23]. From that viewpoint, expr_add.syntax is an instance of na_syntax and expr_add.semantics is an instance of na_semantics. The evidence for the former is provided in lines 2 to 7 in na_syntax. Interestingly, however, our encoding of type classes in Scala is not the common one [24]. In particular, we do not prescribe the use of implicits.

As also announced at the last paragraph of Section III, na_syntax and na_semantics (and also nam_syntax and nam_semantics) relate to the abstract syntax only. This is how they leverage LMS and yet do not suffer from the concrete syntactic anomalies discussed in Section III. Moreover, unlike Modular Reifiable Matching [25], the technique we presented in this section is not exclusively targeting two-level types [26]. The reason is that our technique in this section fully disassociates concrete syntax from the abstract syntax so there no longer is an issue of levels in the types. LMS itself comes with no such separation either - suggesting the name abstract $L M S$ for our technique.

It is noteworthy that the disassociation of abstract and concrete syntax with the lack of the LMS anomalies discussed in Section III needs not specifically be à la LMS. The same impact can also be achieved using integration of a decentralised pattern matching [27]. In the latter technique, the syntax is defined in terms of abstract syntax components. The concrete syntax in the latter technique is then defined on top of those syntax components. The difference is that the abstract LMS composes components (that correspond to ADT cases) additively [28, §17.3], whilst the latter technique would be composing them sequentially.

The connection between this technique and ComponentBased Software Engineering (CBSE) [28, §17],[29, §10] is also interesting. From a CBSE standpoint, nam_syntax is a component in that: Without binding to a particular implementation, it specifies its so-called 'requires' and 'provides' interfaces. The nam_syntax 'requires' interface is its lines 2 and 3 - imposing the following two requirements, respectively: The user of nam_syntax needs to provide a type M. And, there has to be a way to extract two expressions of type $E$ from an instance of M. In return, the 'provides' interface of $M$ is its line 4, where M's Scala match syntax (used in line 8 of nam_semantics) is offered. As such, nam_syntax is promoting the ADT case Mul to its standalone component. ${ }^{1}$ This is an important characteristic of the third technique that relates to the EP. Next section is dedicated to that relationship.

\section{EXPRESSION PROBLEM}

EP is a recurrent problem in the field of Programming Languages, for which a wide range of solutions have thus far been proposed, e.g., [31], [32], [33]. Consider [34], [35], [36], [31], [32], [33], to name a few. Haeri [21] defines EP as the challenge of finding an implementation for an ADT defined by its cases and the functions on it - that:

E1. is extensible in both dimensions, i.e., both new cases and functions can be added.

E2. provides weak static type safety, i.e., applying a function $f$ on a statically ${ }^{2}$ constructed ADT term $t$ should fail to compile when $f$ does not cover all the cases in $t$.

E3. upon extension, forces no manipulation or duplication to the existing code.

E4. accommodates separate compilation, i.e., compiling the extension imposes no requirement for repeating compilation or type checking of existing code. Such static checks should not be deferred to the link or run time.

In Sections II-IV, we presented three techniques for embedded language composition in Scala. All the three techniques satisfy E4. We now reflect on their E1-E3 competence: The first technique clearly satisfies E1. Section III-A2 outlines a scenario where LMS fails to satisfy E1. Whether the third technique satisfies E1 depends on whether it employs trait mixing for composition or not. Note that it needs not. The three techniques all relax E2, although they can be circumvented to work when defaults are available [35]. That is a consequence of Scala performing pattern matching at runtime. LMS too relaxes E2 and that has thus far been considered an acceptable setting. (For example, MVCs [37] and Torgersen's second solution [34] both have the same issue.) The state of affairs for LMS might change in future though [38].

As witnessed by RobotUniExprAddUniDec in Section II-E1, the Scala-unspecific technique fails to satisfy E3 when new cases are to be added. As detailed in Section III-B, LMS has to fight path-dependant typing to satisfy E3 when syntactic categories are updated upon composition. Whether there always is a winning strategy for LMS in such a situation is not known. The third technique clearly satisfies E3.

\footnotetext{
${ }^{1}$ Two reasons for not promoting Num and Add to components: 1) that would complicate presentation. 2) the current design in which those two ADT cases are packed together in a single component (i.e., na_syntax) demonstrates how to address the Common Reuse Principle of Martin [30]

${ }^{2}$ If the guarantee was for dynamically constructed terms too, we would have called it strong static type safety.
} 
We understand that the path-dependant typing difficulties of the LMS-based technique might indeed be a result of our peculiar design. In particular, our choice of giving the syntax and semantics of a language each a trait of their own might be picked as the root cause. We would like to defend that choice of ours, specifically, for the likelihood of engineering (or experimentation with) more than one semantics for the same syntax [15]. In such cases, separation of the syntax and semantics is inevitable.

Finally, one may wonder whether the third technique makes it to a new solution to EP. The answer is indeed yes. At least for EP in presence of defaults [35]. This is the third EP solution of its kind: It promotes ADT cases to their own ADT-parameterised components. See [20], [21] for the first and [27] for the second EP solution of this kind.

\section{RELATED WORK}

a) LISA: As stated earlier, this paper is highly inspired by Mernik [6]. We essentially took his examples for showing how to compose languages embedded in Scala. With LISA being an LDF, even though Scala is famous for its hospitality to embedded languages, we were surprised to end up having less lines-of-code (LoC) in all the three techniques.

Fig. 2 summarises the LoC comparison. In the LoC there, we have also included some syntactic cosmetics that we did not display in this paper. In our experience, the occasions where Scala outperforms LISA by far are those where the task was a ready cake for GPLs. Examples are RobotTime for all the techniques and RobotExtExprAddUniDec for the third technique. For the former, a simple container size query does the job. For the latter, simple trait mixing does.

The first technique generally performs better (in terms of LoC) than LISA. The second is even better usually with its utilisation of trait mixing (dismissing the obvious import s) and super calls. At last, the third is the best with its a posteriori refactoring. The two occasions when LISA considerably outperforms Scala are RobotUniExprAdd for the first technique and RobotUniExprMul for the third. Those correspond to Sections III-A2 and III-B, respectively.

The factored out code in the third technique is not counted in Fig. 2. Once that too is added, the total LoC reaches 328 - which is 2 more than first technique's LoC. We tend to think the reason is the simplicity in the semantics of Mernik's examples. That caused the number of lines the refactoring saves to be less than the extra overhead the technique requires. For more realistic case studies, we expect the balance to be completely different. That would be well in favour of refactoring due to reasonably more involved semantics.

b) Other Language Composition Catalogues: Völter [39] proposes a taxonomy of language composition that he showcases in JetBrains MPS. His taxonomy is along axes, not all of which having a clear correspondent in the work of Erdweg et al. As explained by Mernik, the resulting ways for language composition that Völter prescribes, however, are subsumed by the latter taxonomy. Völter's taxonomy gives (syntax-oriented) IDE development for languages a higher weight.
Barrett, Bolz, and Tratt [40] catalogue composition of six different Python and Prolog virtual machines. Their study has a particular focus on measuring performance of the resulting interpreters upon composition.

Zhang et al. [41] facilitate composition of languages that are embedded using Object Algebras [42]. This is achieved using their simple predesignated annotation. Their showcase focuses on hierarchies of language extension. Using linearised multiple language inheritance, they also simulate a single language unification. Zhang et al. do not consider higher order composition.

Melange [43] is an LDF that is specially equipped for language composition. Various syntactic facilities are available in Melange to instruct mix-and-match for many different aspects of a language - ranging from syntax, dynamic and static semantics, and name-binding to IDE features. Language composition under Melange is catalogued for a small set of showcases but with in-length discussions on customisability. The current documentation of Melange, however, makes it hard for us to compare its catalogue of language composition with similar works. Specifically, we fail to figure out which ways for language composition Melange supports in general (namely, for other scenarios than the ones already in their documentation) and how.

c) Components for Language Specification: PLanCompS funcons are syntactic constructs that ship with their own fixed static and dynamic semantics (presented in MSOS). The PLanCompS specification of a programming language is developed by merely assembling funcons [44]. Example assemblies are larger academic languages [45] and mediumscale ones [46]. Despite their merit, funcons do not constitute CBSE components. In particular, funcons do not ship with their 'requires' interfaces.

MVCs [37] are components for solving an extension to EP. Rather than components in their CBSE sense, however, MVCs are components in a Component-Oriented Programming [47] sense. (Cf. [21, §4.3].) MVCs rely on the implementation details of how a component realises its interfaces. CBSE components, in contrast, are identified by their 'requires' and 'provides' interfaces.

Haeri and Schupp [20], [27] take a CBSE approach for the implementation of embedded languages. Their approach employs type constraints and multiple inheritance. The third technique here employs (possibly constrained) abstract types instead of type parameters. Although essentially the same, the former can make code terser. In Scala, however, offering the match syntax is apparently not possible for type parameters.

Finally, Cazzola and Vacchi [48] too have taken a CBSE approach. Their components correspond to a DSL's compiler passes. Accordingly, how their work relates to the common language specification formalisms is not clear. In contrast, components in our third technique are ADT cases - acting as the unit of study for formal semantics.

d) Component-Based AGs: AGs are a powerful means for language specification with many benefits that are wellstudied. Attempts to modularise AGs go back to Saraiva and 


\begin{tabular}{c|cccccccccccccc} 
& $L_{1}$ & $L_{2}$ & $L_{3}$ & $L_{4}$ & $L_{5}$ & $L_{6}$ & $L_{7}$ & $L_{8}$ & $L_{9}$ & $L_{10}$ & $L_{11}$ & $L_{12}$ & $L_{13}$ & Sum \\
\hline LISA & 42 & 23 & 13 & 19 & 19 & 32 & 39 & 41 & 20 & 34 & 23 & 20 & 19 & 344 \\
$T_{1}$ & 32 & 7 & 16 & 26 & 34 & 11 & 40 & 25 & 31 & 34 & 17 & 22 & 31 & 326 \\
$T_{2}$ & 30 & 6 & 15 & 20 & 29 & 10 & 34 & 20 & 26 & 28 & 13 & 23 & 33 & 287 \\
$T_{3}$ & 29 & 5 & 15 & 16 & 16 & 10 & 10 & 20 & 23 & 6 & 13 & 23 & 16 & 202
\end{tabular}

Columns: $L_{1}=$ Robot, $L_{2}=$ RobotTime, $L_{3}=$ RobotPositive, $L_{4}=$ ExprAdd, $L_{5}=$ RobotUniExprAdd, $L_{6}=$ Dec, $L_{7}=$ RobotUniExprAddUniDec, $L_{8}=$ RobotTimeSpeed, $L_{9}=$ ExprAddUniDec, $L_{10}=$ RobotExtExprAddUniDec, $L_{11}=$ RobotUniExprAddExtRobotTime, $L_{12}=$ ExprMul, $L_{13}=$ RobotUniExprMul Rows: LISA = Mernik's Implementation, $T_{i}=$ Technique $i$, for $i \in\{1,2,3\}$

Fig. 2: Lines-of-Code Comparison between Mernik's LISA and Our Three Techniques

Swierstra [49]. Saraiva's Higher Order AGs (HOAGs) [50] were the initial steps towards using AGs in a componentbased fashion. Viera and Swierstra [51] formally define several ways to combine HOAGs. However, those ways do not tightly correspond to the usual composition mechanics of generalpurpose languages.

So long as EP is concerned, the correct behaviour of a HOAG w.r.t. E2 is not universally agreed upon. In terms of HOAGs, that amounts to the absence of an attribute expected from another component in the mix. In particular, should the code then fail statically or dynamically? Zipper functions [52], [53] act like HASKELL by statically reporting such errors so long as they can be caught iteratively [54].

Kiama [55] uses AGs embedded in Scala for language specification. It is possible to use Kiama in a component-based fashion - as done for embedding Oberon-0 [56] in Scala [57]. However, disassociation of the concrete and abstract syntax can become non-trivial in Kiama. We anticipate that would cause similar difficulties to those we faced over our second technique. For the Oberon-0 embedding, facing such difficulties were unlikely for the different pieces of syntax were all available in advance. On the contrary, whilst composing unrelated pieces of syntax, clash of concrete syntax is likely.

\section{CONCLUSIONS AND FUtURE WORK}

In this paper we present three different techniques for composing languages embedded in Scala. The first is Scalaunspecific and works in presence of common module systems and higher order functions (Section II). The second is LMSbased and requires mixin composition and super calls (Section III). The third works by promoting ADT cases to ADTparameterised components (Section IV). We showcase the three techniques using the example compositions of Mernik, which, in return, were designed to exhibit LISA's composition facilities for Erdweg et al.'s taxonomy of composition. We manifest the strengths and weaknesses of each technique. We compare them according to their performance as EP solutions (Section V) and LoC (Section VI-0a).

Systematic study of embedded language composition is a young topic. Numerous paths exist for future research. Examining our third technique against larger testcases is an immediate future work. A promising candidate is the LDTA'11 challenge of modular implementation of Oberon0 . The testcase can then be compared with the LDTA'11 contestants. We anticipate complications in dealing with a few issues along the way: Firstly, the technique takes a design-bycontract approach on the names it chooses for abstract types, e.g., $\mathrm{A}$ and $\mathrm{N}$ in na_syntax. In large scale, these names are likely to clash upon composition. Avoiding that would imply a priori knowledge. That kind of knowledge is, however, rare in experimental language design. Secondly, outside lab settings, usual software engineering techniques may become inevitable. We took the lab liberty of not being concerned with that here. For example, position and consts lack proper scoping and are common intact amongst all the descendants of Robot and Dec, respectively.

Type classes are more widely practised in HASKELL. It would be interesting to see our third technique in HASKELL with its type classes instead of Scala's mixins and inheritance. The comparison between the results of ours and those according to the following two HASKELL EP solutions would be particularly interesting: Data Types a la Carte [36] and Parametric Compositional Datatypes [32].

Object Algebras are gaining gravity as a powerful abstraction for embedded language development [31], [58], [59], [41]. The current technology for embedding Object Algebras, however, is heavyweight in both term creation [60] and algebra composition. It is easy to turn na_syntax and the like into Object Algebra Interfaces to lower those two weights. How useful the result would be in lowering those two weights in the current Object Algebras technology is another future work.

Finally, it is important to also produce catalogues like this paper in other host languages than Scala. Many languages have merits in hosting other languages. But, the limits of that and the key factors of it are not clear. Composition of the embedded languages is certainly amongst the important factors. A head-to-head comparison on hospitality of language composition is missing. We are currently working on that.

\section{REFERENCES}

[1] S. Erdweg, P. G. Giarrusso, and T. Rendel, "Language Composition Untangled," in $12^{\text {th }}$ LDTA, A. Sloane and S. Andova, Eds. ACM, Mar. 2012, p. 7.

[2] T. Rompf and M. Odersky, "Lightweight Modular Staging: a Pragmatic Approach to Runtime Code Generation and Compiled DSLs," in $9^{\text {th }}$ GPCE. Eindhoven, Holland: ACM, 2010, pp. 127-136.

[3] W. R. Cook, "Object-Oriented Programming Versus Abstract Data Types," in FOOL, ser. LNCS, J. W. de Bakker, W. P. de Roever, and G. Rozenberg, Eds., vol. 489, Holland, Jun. 1990, pp. 151-178.

[4] J. C. Reynolds, "User-Defined Types and Procedural Data Structures as Complementary Approaches to Type Abstraction," in New Direc. Algo. Lang., S. A. Schuman, Ed. INRIA, 1975, pp. 157-168.

[5] P. Wadler, "The Expression Problem," Nov. 1998, Java Genericity Mailing List. 
[6] M. Mernik, "An Object-Oriented Approach to Language Compositions for Software Language Engineering,” J. Sys. \& Soft., vol. 86, no. 9, pp. 2451-2464, 2013.

[7] M. Mernik, M. Lenic, E. Avdicausevic, and V. Zumer, "LISA: An Interactive Environment for Programming Language Development," in $11^{\text {th }} C C$, ser. LNCS, R. N. Horspool, Ed., vol. 2304. Springer, Apr. 2002, pp. 1-4

[8] D. E. Knuth, "Semantics of Context-Free Languages," Math. Sys. Theo. vol. 2 , no. 2 , pp. $127-145,1968$

[9] J. Paakki, "Attribute Grammar Paradigms - A High-Level Methodology in Language Implementation," ACM Comp. Surv., vol. 27, no. 2, pp. 196-255, 1995.

[10] P. Trinder, K. Hammond, H.-W. Loidl, and S. Peyton Jones, "Algorithm + Strategy = Parallelism,” JFP, vol. 8, no. 1, pp. 23-60, Jan. 1998.

[11] A. Dijkstra, J. Fokker, and S. D. Swierstra, "The Architecture of the Utrecht Haskell Compiler," in $2^{\text {nd }}$ Haskell, S. Weirich, Ed Edinburgh, Scotland: ACM, 2009, pp. 93-104.

[12] M. Odersky, "Pimp my Library," Artima Developer Blog, vol. 9, Oct. 2006.

[13] S. H. Haeri and S. Schupp, "Expression Compatibility Problem," in $7^{\text {th }}$ SCSS, ser. EPiC Comp., J. H. Davenport and F. Ghourabi, Eds., vol. 39. EasyChair, Mar. 2016, pp. 55-67.

[14] J. Jeuring, S. Leather, J. P. Magalhães, and A. R. Yakushev, "Libraries for Generic Programming in HASKell," in Adv. Func. Prog., $6^{\text {th }}$ Int. School, AFP, ser. LNCS, P. W. M. Koopman, R. Plasmeijer, and S. D. Swierstra, Eds., vol. 5832. Springer, May 2008, pp. 165-229.

[15] C. Hofer, K. Ostermann, T. Rendel, and A. Moors, "Polymorphic Embedding of DSLs," in $7^{\text {th }}$ GPCE, Y. Smaragdakis and J. G. Siek, Eds. Nashville, TN, USA: ACM, Oct. 2008, pp. 137-148.

16] M. Odersky and M. Zenger, "Scalable Component Abstractions," in 20 th OOPSLA. San Diego, CA, USA: ACM, 2005, pp. 41-57.

[17] E. Ernst, "Family Polymorphism," in $15^{t h}$ ECOOP, ser. LNCS, J. Lindskov Knudsen, Ed., vol. 2072. Springer, Jun. 2001, pp. 303-326.

[18] C. Saito, A. Igarashi, and M. Viroli, "Lightweight Family Polymorphism," J. Func. Prog., vol. 18, no. 3, pp. 285-331, 2008.

[19] M. Fowler, "Refactoring: Improving the Design of Existing Code," in $2^{\text {nd }}$ XP/Agile, ser. LNCS, D. Wells and L. A. Williams, Eds., vol. 2418. Springer, Aug. 2002, p. 256.

[20] S. H. Haeri and S. Schupp, "Reusable Components for Lightweight Mechanisation of Programming Languages," in $12^{t h} S C$, ser. LNCS W. Binder, E. Bodden, and W. Löwe, Eds., vol. 8088. Springer, Jun. 2013, pp. 1-16.

[21] S. H. Haeri, "Component-Based Mechanisation of Programming Languages in Embedded Settings," Ph.D. dissertation, STS, TUHH, Germany, Dec. 2014.

[22] P. D. Mosses, "Modular Structural Operational Semantics," JLAP, vol. 60-61, pp. 195-228, 2004

[23] P. Wadler and S. Blott, "How to Make ad-hoc Polymorphism Less adhoc," in $16^{\text {th }}$ POPL. ACM Press, Jan. 1989, pp. 60-76.

[24] B. C. d. S. Oliveira, A. Moors, and M. Odersky, "Type Classes as Objects and Implicits," in $25^{\text {th }}$ OOPSLA, W. R. Cook, S. Clarke, and M. C. Rinard, Eds. ACM, Oct. 2010, pp. 341-360.

[25] B. C. d. S. Oliveira, S.-C. Mu, and S.-H. You, "Modular Reifiable Matching: A List-of-Functors Approach to Two-Level Types," in $8^{\text {th }}$ HASKELl, B. Lippmeier, Ed. ACM, Sep. 2015, pp. 82-93.

[26] T. Sheard and E. Pasalic, "Two-Level Types and Parameterized Modules," JFP, vol. 14, no. 5, pp. 547-587, 2004

[27] S. H. Haeri and S. Schupp, "Integration of a Decentralised Pattern Matching: Venue for a New Paradigm Intermarriage," in $8^{\text {th }}$ SCSS, ser. EPiC Comp., M. Mosbah and M. Rusinowitch, Eds., vol. 45. EasyChair, Apr. 2017, pp. 16-28

[28] I. Sommerville, Software Engineering, $9^{\text {th }}$ ed. Addison-Wesley, 2011.

[29] R. S. Pressman, Software Engineering: A Practitioner's Approach, $7^{\text {th }}$ ed. McGraw-Hill, 2009.

[30] R. C. Martin, "Design Principles and Design Patterns," 2000, online article available from the ObjectMentor website.

[31] B. C. d. S. Oliveira and W. R. Cook, "Extensibility for the Masses - Practical Extensibility with Object Algebras," in $26^{\text {th }}$ ECOOP, ser. LNCS, vol. 7313. Springer, 2012, pp. 2-27.

[32] P. Bahr and T. Hvitved, "Parametric Compositional Data Types," in $4^{\text {th }}$ $M S F P$, ser. ENTCS, J. Chapman and P. B. Levy, Eds., vol. 76, Feb. 2012, pp. 3-24.

[33] Y. Wang and B. C. d. S. Oliveira, "The Expression Problem, Trivially!" in $15^{\text {th }}$ Modularity. New York, NY, USA: ACM, 2016, pp. 37-41.
[34] M. Torgersen, "The Expression Problem Revisited" in $18^{\text {th }}$ ECOOP, ser. LNCS, M. Odersky, Ed., vol. 3086, Oslo (Norway), Jun. 2004, pp. 123-143.

[35] M. Odersky and M. Zenger, "Independently Extensible Solutions to the Expression Problem," in FOOL, Jan. 2005.

[36] W. Swierstra, "Data Types à la Carte," JFP, vol. 18, no. 4, pp. 423-436, 2008.

[37] B. C. d. S. Oliveira, "Modular Visitor Components," in $23^{\text {rd }}$ ECOOP, ser. LNCS, vol. 5653. Springer, 2009, pp. 269-293.

[38] T. Rompf, "Reflections on LMS: Exploring Front-End Alternatives," in $7^{\text {th }}$ SIGPLAN Symp. Scala, A. Biboudis, M. Jonnalagedda, S. Stucki, and V. Ureche, Eds. ACM, Nov. 2016, pp. 41-50.

[39] M. Völter, "Language and IDE Modularization and Composition with MPS," GTTSE, vol. 7680, pp. 383-430, 2011.

[40] E. Barrett, C. F. Bolz, and L. Tratt, "Approaches to Interpreter Composition," Comp. Lang., Sys. \& Struct., vol. 44, pp. 199-217, 2015.

[41] H. Zhang, Z. Chu, B. C. d. S. Oliveira, and T. van der Storm, "Scrap Your Boilerplate with Object Algebras," in $29^{\text {th }}$ OOPSLA, J. Aldrich and P. Eugster, Eds., Oct. 2015, pp. 127-146.

[42] Guttag, J. V. and Horning, J. J., "The Algebraic Specification of Abstract Data Types," Acta Informatica, vol. 10, pp. 27-52, 1978

[43] T. Degueule, B. Combemale, A. Blouin, O. Barais, and J.-M. Jézéquel, "Melange: A Meta-Language for Modular and Reusable Development of DSLs," in $8^{\text {th }} S L E$, R. F. Paige, D. Di Ruscio, and M. Völter, Eds., Oct. 2015, pp. 25-36.

[44] P. D. Mosses, "Component-Based Description of Programming Languages," in BCS Int. Acad. Conf., E. Gelenbe, S. Abramsky, and V. Sassone, Eds. Brit. Comp. Soc., 2008, pp. 275-286.

[45] P. D. Mosses and F. Vesely, "FunKons: Component-Based Semantics in $\mathbb{K}$," in WRLA, ser. LNCS, S. Escobar, Ed., vol. 8663. Springer, Apr. 2014.

[46] M. Churchill, P. D. Mosses, N. Sculthorpe, and P. Torrini, "Reusable Components of Semantic Specifications," Trans. Aspect-Orient. Soft. Dev. XII, vol. 12, pp. 132-179, 2015.

[47] M. D. McIlroy, "Mass Produced Software Components," in Proc. NATO Conf. Soft. Eng. New York, US: Petrocelli/Charter, 1969, pp. 138-155.

[48] W. Cazzola and E. Vacchi, "Language Components for Modular DSLs using Traits," ComLan, vol. 45, pp. 16 - 34, 2016.

[49] J. Saraiva and D. Swierstra, "Generic Attribute Grammars," in $2^{\text {nd }}$ WAGA, vol. 99, 1999, pp. 185-204.

[50] J. Saraiva, "Component-Based Programming for Higher-Order Attribute Grammars," in $1^{\text {st }}$ GPCE, ser. LNCS, D. S. Batory, C. Consel, and W. Taha, Eds., vol. 2487. Springer, Oct. 2002, pp. 268-282.

[51] M. Viera and D. Swierstra, "Attribute Grammar Macros," Sci. Comp. Prog., vol. 96, pp. 211-229, 2014.

[52] P. Martins, J. P. Fernandes, J. Saraiva, E. Van Wyk, and A. Sloane, "Embedding Attribute Grammars and their Extensions using Functional Zippers," Sci. Comp. Prog., vol. 132, pp. 2-28, 2016.

[53] J. P. Fernandes, P. Martins, A. Pardo, J. Saraiva, and M. Viera, "Memoized Zipper-Based Attribute Grammars and their Higher Order Extension," Sci. Comp. Prog., vol. 173, pp. 71-94, 2019

[54] A. Middelkoop, A. Dijkstra, and D. Swierstra, "Iterative Type Inference with Attribute Arammars," in $9^{\text {th }}$ GPCE, E. Visser and J. J., Eds. ACM, Oct. 2010, pp. 43-52.

[55] A. M. Sloane, "Lightweight Language Processing in Kiama," in GTTSE III, ser. LNCS, J. M. Fernandes, R. Lämmel, J. Visser, and J. Saraiva, Eds., vol. 6491. Springer, Jul. 2009, pp. 408-425.

[56] N. Wirth, Compiler Construction, ser. Int. Comp. Sci. Series. AddisonWesley, 1996.

57] A. M. Sloane and M. Roberts, "Oberon-0 in Kiama," Sci. Comp. Prog. vol. 114 , pp. 20-32, 2015

[58] B. C. d. S. Oliveira, T. van der Storm, A. Loh, and W. R. Cook, "FeatureOriented Programming with Object Algebras," in $27^{\text {th }}$ ECOOP, ser LNCS, G. Castagna, Ed., vol. 7920. Montpellier, France: Springer, 2013, pp. 27-51

[59] T. Rendel, J. I. Brachthäuser, and K. Ostermann, "From Object Algebras to Attribute Grammars," in $28^{\text {th }}$ OOPSLA, A. P. Black and T. D. Millstein, Eds. ACM, Oct. 2014, pp. 377-395.

[60] A. P. Black, "The Expression Problem, Gracefully," in MASPEGHI@ECOOP 2015, M. Sakkinen, Ed. ACM, Jul. 2015, pp. $1-7$. 